\title{
Fluorescence monitoring of rare circulating tumor cell and cluster dissemination in a multiple myeloma xenograft model in vivo
}

Roshani Patil

Xuefei Tan

Peter Bartosik

Alexandre Detappe

Judith M. Runnels

Irene Ghobrial

Charles P. Lin

Mark Niedre 


\title{
Fluorescence monitoring of rare circulating tumor cell and cluster dissemination in a multiple myeloma xenograft model in vivo
}

\author{
Roshani Patil, ${ }^{\mathrm{a}, \dagger}$ Xuefei Tan, ${ }^{\mathrm{b}, \dagger}$ Peter Bartosik, ${ }^{\text {a }}$ Alexandre Detappe, ${ }^{\mathrm{c}}$ Judith M. Runnels, ${ }^{\mathrm{d}}$ Irene Ghobrial, ${ }^{\mathrm{c}}$ \\ Charles P. Lin, ${ }^{d}$ and Mark Niedre ${ }^{a, b, *}$ \\ aNortheastern University, Department of Bioengineering, Boston, Massachusetts, United States \\ ${ }^{b}$ Northeastern University, Department of Electrical and Computer Engineering, Boston, Massachusetts, United States \\ 'Dana Farber Cancer Institute, Harvard Medical School, Department of Medical Oncology, Boston, Massachusetts, United States \\ dMassachusetts General Hospital and Harvard Medical School, Center for Systems Biology and Wellman Center for Photomedicine, Boston, \\ Massachusetts, United States
}

\begin{abstract}
Circulating tumor cells (CTCs) are of great interest in cancer research because of their crucial role in hematogenous metastasis. We recently developed "diffuse in vivo flow cytometry" (DiFC), a preclinical research tool for enumerating extremely rare fluorescently labeled CTCs directly in vivo. In this work, we developed a green fluorescent protein (GFP)-compatible version of DiFC and used it to noninvasively monitor tumor cell numbers in circulation in a multiple myeloma (MM) disseminated xenograft mouse model. We show that DiFC allowed enumeration of CTCs in individual mice overtime during MM growth, with sensitivity below $1 \mathrm{CTC} \mathrm{mL}^{-1}$ of peripheral blood. DiFC also revealed the presence of CTC clusters (CTCCs) in circulation to our knowledge for the first time in this model and allowed us to calculate CTCC size, frequency, and kinetics of shedding. We anticipate that the unique capabilities of DiFC will have many uses in preclinical study of metastasis, in particular, with a large number of GFP-expressing xenograft and transgenic mouse models. $\odot$ The Authors. Published by SPIE undera Creative Commons Attribution 4.0 Unported License. Distribution or reproduction of this work in whole or in part requires full attribution of the original publication, including its DOI. [DOI: 10.1117/1.JBO.24.8.085004]
\end{abstract}

Keywords: optical devices; fluorescence; circulating tumor cells; circulating tumor cell clusters; multiple myeloma.

Paper 190086R received Mar. 26, 2019; accepted for publication Aug. 5, 2019; published online Aug. 27, 2019.

\section{Introduction}

Metastasis is responsible for over $90 \%$ of cancer-related deaths, and the most common pathway is via the peripheral blood. The presence and number of circulating tumor cells (CTCs) are known to be associated with prognosis for many cancer types. ${ }^{1,2}$ As such, CTCs are a major focus of clinical and preclinical research. ${ }^{3-5}$ CTCs are exceedingly rare-fewer than $1 \mathrm{CTC} \mathrm{mL}^{-1}$ of peripheral blood is associated with poor overall survival. Enumeration of CTCs is usually performed by drawing and analyzing peripheral blood samples, wherein target cells are isolated using methods including flow cytometry (FC), size-based cell separation, immunomagnetic separation, and microfluidic capture. ${ }^{6}$ In vivo cell-capture techniques, such as functionalized medical wires, have also been developed for enumeration of CTCs. ${ }^{7,8}$

Although "liquid biopsy" tools remain the workhorse of preclinical CTC research, they have a number of known limitations, particularly in the context of longitudinal study of animals over time: ${ }^{9,10}$ for survival experiments, blood collection is limited to about $10 \%$ of the peripheral blood volume every 2 weeks, ${ }^{11}$ which may result in significant inaccuracies in estimating the CTC burden due to sampling error. ${ }^{12,13}$ In some cases involving rare cells, mice must be euthanized so that the entire peripheral blood volume may be analyzed. Moreover, limited temporal sampling makes it difficult to observe the kinetics of CTC shedding, which may vary significantly over the timescale of days

*Address all correspondence to Mark Niedre, E-mail: m.niedre@neu.edu

†These authors contributed equally to this work. and (as we show) even minutes. Blood samples are also known to degrade rapidly after removal from the body, ${ }^{14}$ and the process of drawing blood can trigger a stress response in the animal. $^{15}$

In addition to single CTCs, CTC clusters (CTCCs, also called circulating tumor microemboli, $\mathrm{CTM}^{16}$ ) are multicellular groupings of CTCs that exhibit a mixture of epithelial and mesenchymal properties and may include stromal ${ }^{17}$ and other nontumor cells. ${ }^{18,19}$ CTCCs are even rarer than individual CTCs but are known to have significantly better survivability in circulation and higher metastatic potential ( $\sim 50$-fold). Much is still not understood about CTCCs, including the mechanisms for their survival advantage in vivo, as well as their abundance, dynamics of shedding, and composition. It has been suggested that a key reason for this is that nearly all CTC isolation methods are not designed to detect clusters and therefore may result in their dissolution or loss. ${ }^{18}$ This has also driven the development of microfluidic systems specifically designed to isolate clusters in recent years. ${ }^{20}$

Our team recently developed a small animal research tool, "diffuse in vivo flow cytometry" (DiFC). ${ }^{21} \mathrm{DiFC}$ is a variant of "in vivo flow cytometry" (IVFC), which is a general class of instruments for counting circulating cells in the bloodstream without drawing blood samples using fluorescence or photoacoustic technologies. ${ }^{9,10,22,23}$ The distinguishing feature of DiFC compared to other IVFC methods is that it uses diffuse light to detect fluorescently labeled circulating cells in large blood vessels such as the tail ventral caudal artery (VCA) in mice. DiFC uses a dual-optical probe configuration that is placed on the skin surface approximately over a large blood 
vessel. We previously showed that DiFC allows interrogation of hundreds of microliters of circulating blood per minute in mice. ${ }^{21}$ By contrast, microscopy-IVFC methods sample small blood vessels in the mouse ear, where blood flow rates are on the order of $1 \mu \mathrm{L} \mathrm{min}{ }^{-1} .{ }^{10}$ In our previous work, DiFC used near-infrared (NIR) fluorescent dyes because of the minimal attenuation of NIR light in biological tissue. ${ }^{24}$ However, this limited the use of DiFC to cells expressing NIR fluorescent proteins (FPs), ${ }^{25}$ which are significantly less common in biomedical research than visible FPs, such as the green fluorescent protein (GFP). ${ }^{10,15,26}$

The novelty of the present work is threefold. First, we developed a blue-green version of DiFC suitable for use with GFP, thereby greatly expanding the utility of the method. Second, we used our GFP-DiFC system to monitor the growth and vascular spread of multiple myeloma (MM) in a disseminated xenograft model (DXM) in mice. ${ }^{27} \mathrm{MM}$ is a hematological malignancy that is believed to originate at a single site in the bone marrow niche and then continuously disseminate by the circulatory system. Hence, MM is of significant interest to researchers both as a disease and as a model of cancer metastasis. ${ }^{28}$ Third, DiFC revealed evidence for the presence of MM CTCCs in circulation in this model to our knowledge for the first time. Overall, these experiments are extremely difficult to perform with liquid biopsy methods and illustrate the value of DiFC as a complementary method in the study of hematogenous metastasis.

\section{Methods and Materials}

\subsection{DiFC Instrument}

The GFP-DiFC instrument schematic is shown in Fig. 1(a). The system is similar to our previously reported NIR DiFC system ${ }^{21}$ but uses optical components compatible with GFP. Specifically, the light source was 488-nm DPSS laser (DL488-150; Crystalaser LLC, Nevada), the output of which was filtered with a cleanup band-pass (BP-x) with 488/10 nm (ZET488/10x; Chroma Technology Corporation, Bellows Falls, Vermont). These were coupled into the source fiber of the fiber probes using lens-fiber couplers (FC-x) with 532-nm antireflection coating (F240SMA-532; Thorlabs Inc., Newton, New Jersey). The fiber bundles were custom designed and built for this application (EMVision LLC, Loxahatchee, Florida). Two filters were mounted directly to the tip of the fiber bundles, which we found necessary to mitigate fiber autofluorescence [Fig. 1(a), inset]. These were a central band-pass filter (BP-f) at 488/5 $\mathrm{nm}$ to filter

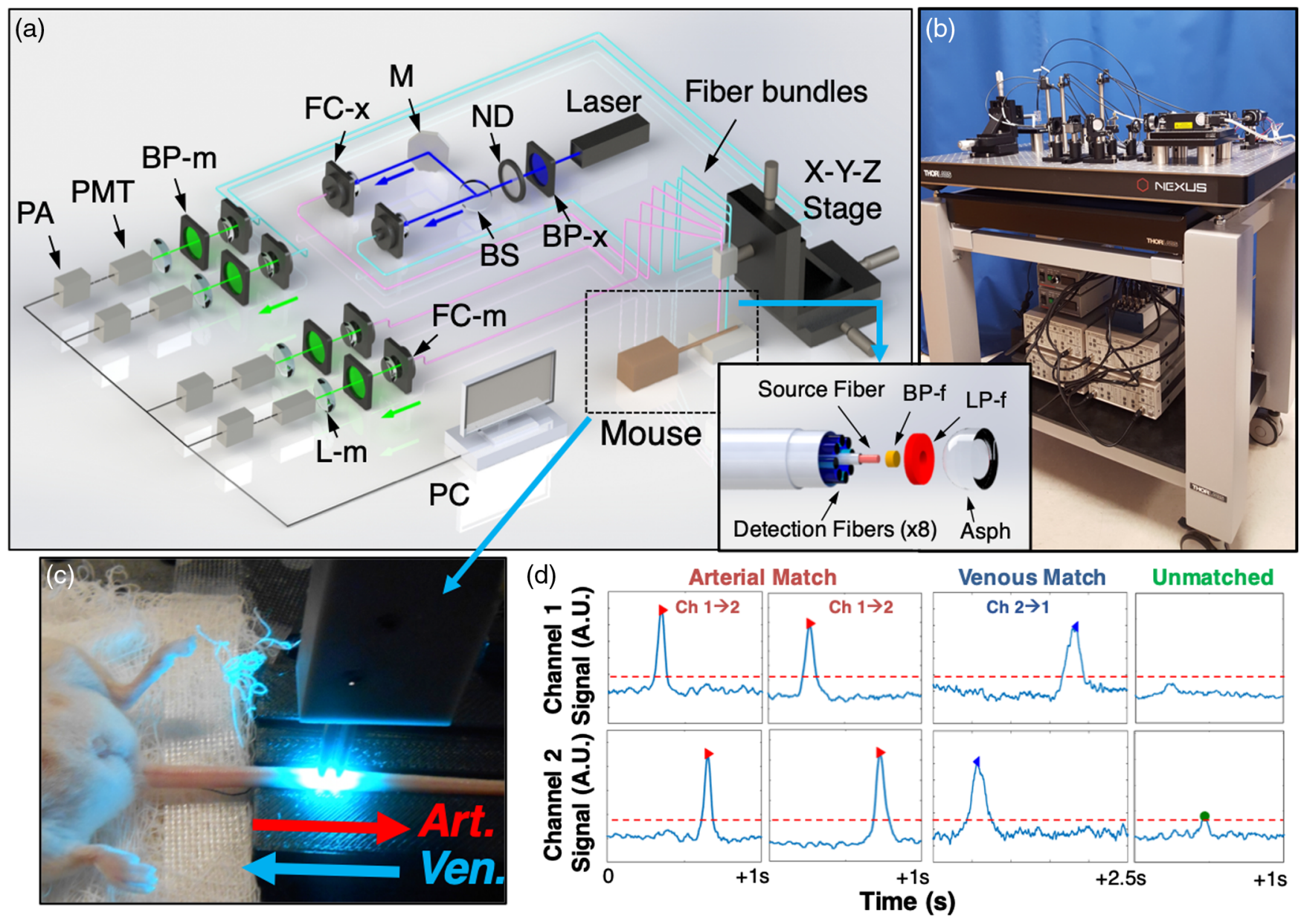

Fig. 1 (a) GFP-DiFC instrument schematic and fiber probe design (inset; see text for details). (b) The DiFC system was mounted on an optics cart that could be moved easily between sites. (c) The fiber bundles were placed on the ventral surface of the mouse tail approximately above the ventral caudal bundle. (d) DiFC allows detection and discrimination of circulating MM GFP-labeled CTCs moving the arterial and venous directions. Peaks that were not measured in both channels were assumed to be moving in smaller blood vessels or due to noise, and were subsequently discarded from the analysis. 
the excitation light and an outer detection ring shape long-pass filter (LP-f) at $503 \mathrm{~nm}$ for collection of fluorescence light. The output of the collection fiber bundles (eight per probe) was terminated on a second set of lens fiber couplers (FC-m) and then filtered with interference filters (BP-m) at 535/50 nm (ET535/50 m; Chroma). The band-pass filters were identical here but could be different in the future to allow multispectral measurement of GFP and a second fluorophore simultaneously. Four current-output photomultiplier tubes (PMTs; H6780-20, Hamamatsu Photonics, Bridgewater, New Jersey) were powered by a voltage supply (C10709, Hamamatsu). The output of each PMT was amplified with low-noise current preamplifiers (PA; SR570, Stanford Research Systems, Sunnyvale, California) with $300 \mathrm{~Hz}$ low-pass filter and then digitized with a multifunction data acquisition board (USB-6212 BNC; National Instruments, Austin, Texas). The entire setup was mounted on an optics cart (POC001, Thorlabs) so that it could be moved easily between sites [Fig. 1(b)].

\subsection{DiFC Data Analysis}

GFP-DiFC data were analyzed, as we described previously, ${ }^{21}$ with a few minor differences. Data were first preprocessed by summing the data from the two PMTs per channel, performing background subtraction, and then applying a 5-ms moving average filter.

\subsubsection{CTC detection}

Data from the two channels were analyzed using a two-step procedure. In the first step, "single cell candidates" were detected in the data using a threshold of $75 \mathrm{nA}$ (this was empirically selected to yield a low FAR). In the second step, candidates were matched in either the forward or reverse directions, according to similarities in the peak width, amplitude, and temporal separation (between the two fibers) in relation to the estimated cell speed. This allowed us to distinguish cells moving the forward (arterial) and reverse (venous) directions and to distinguish "unmatched" peaks as false alarm signals due to electronic noise [Fig. 1(d)]. This also removed motion artifacts, which occasionally occurred, since these resulted in false-peaks that were detected both channels simultaneously, and thus were excluded based on the matching criteria.

\subsubsection{CTCC detection}

As we discuss in more detail below, an important result in this manuscript versus our previous work ${ }^{21}$ is the detection of CTCC-like signals, which were characterized as having large amplitude and width compared to individual cells, implying the presence of cell groupings. Unlike the signals from single cells, there were frequently relatively lengthy delays (seconds or minutes) between detections on the two DiFC channels or no obvious second detection. As such, "matching" between channels was difficult, so the detection criterion we used for CTCCs was based on amplitude and temporal width alone-specifically, with amplitude exceeding $300 \mathrm{nA}$, and full-width at halfmaximum (FWHM) exceeding $100 \mathrm{~ms}$. These criteria resulted no false-alarm detections in our control mice. We reported the average detection rate per DiFC channel per minute. We also estimated the equivalent number of cells in a cluster by dividing the amplitude of the cluster by the mean amplitude of a single cell.

\subsection{Phantom Experiments in Vitro}

As an initial test of the GFP-DiFC system, we used a flow phantom model similar to our previous work. ${ }^{29}$ Briefly, the "flow phantom" was a block of optically diffusing high-density polyethylene with an embedded strand of microbore Tygon tubing (TGY-010-C, Small Parts, Inc., Seattle, Washington) at a depth of $0.5 \mathrm{~mm}$. The tubing was connected to a syringe pump (70-2209, Harvard Apparatus, Holliston, Massachusetts). We pumped suspensions of Dragon Green reference intensity 4 (DG4) microspheres (Bangs Laboratories Inc., Fisher, Indiana) through the tubing. DG microspheres are $6 \mu \mathrm{m}$ diameter fluorescence standards with absorption and emission spectra similar to GFP. We used suspensions of 500 DG4 spheres $\mathrm{mL}^{-1}$ of PBS, with a flow speed of $30 \mu \mathrm{L} \mathrm{min}{ }^{-1}$. PBS-only was used as controls.

\subsection{Multiple Myeloma Disseminated Xenograft Model in Mice}

All mice were handled in accordance with Northeastern University's Institutional Animal Care and Use Committee (IACUC) policies on animal care. Animal experiments were carried out under Northeastern University IACUC protocol \#15-0728R. All experiments and methods were performed with an approval from and in accordance with relevant guidelines and regulations of Northeastern University IACUC.

Mice were held under inhaled isofluorane during DiFC scanning to prevent movement and were kept warm using two heating pads placed under the body and over the exposed area of the tail. The tail was placed in a custom 3-D printed holder to mitigate any breathing movement while under anesthetic. The DiFC fiber probes were separated by $3 \mathrm{~mm}$ and were placed firmly in contact with the skin, approximately over the large vascular bundle on the ventral side of the tail [Fig. 1(c)]. Mice were scanned with DiFC twice per week for $45 \mathrm{~min}$ at 2000 samples s$^{-1}$. The first 10 min of acquisition data was discarded since we often observed transient effects, which we attributed to the warming of the tail with the heating pad (i.e., a transient increase in blood flow and CTC count rate).

We used MM.1S MM cells that were genetically modified to carry GFP, firefly luciferase, and neomycin genes (MM. 1S.GFP.Luc cells). These cells were originally described by Dr. Rosen at Northwestern University. Cells were authenticated by an external service (Bio-Synthesis Inc., Lewisville, Texas) to verify their MM.1S lineage.

We used 8-week-old male severe combined immunodeficient (SCID)/Bg mice (Charles River) tail vein injected (i.v.) with $5 \times$ $10^{6}$ MM.1S.GFP.Luc cells suspended in $200 \mu \mathrm{L}$ PBS $(N=8)$ or PBS-injected controls $(N=4)$. SCID mice required removal of the hair on the tail region with depilatory cream (Nair). We also used clear imaging gel (Ultrasound and Laser Gel \#4963, McKesson Medical-Surgical Inc., Richmond, Virginia) applied to the skin surface to facilitate optical coupling. Control mice were always scanned on the same day as MM-bearing mice, to account for the possibility that the background noise properties of the instrument may change day-to-day.

Mice were grown in two separate cohorts of $N=4$ tumor bearing and $N=2$ control each. For reasons described in detail below, for the first cohort ("cohort 1"; C1), mice were followed up to 31 days after injection. For the second cohort ("cohort 2"; $\mathrm{C} 2$ ), mice were followed up to 36 days after injection. 


\subsection{Bioluminescence Imaging}

As a secondary method for tracking MM growth, bioluminescence imaging (BLI) was performed weekly with a commercial IVIS Lumina II imaging system (Caliper Life Sciences, now Perkin Elmer, Waltham, Massachusetts). Mice were injected i.p. with $150 \mathrm{mg} \mathrm{kg}^{-1}$ of D-luciferin (Perkin Elmer) 10 min prior to imaging. The image exposure time was $60 \mathrm{~s}$.

\subsection{Flow Cytometry}

For mice in $\mathrm{C} 1$, we drew blood samples for analysis with $\mathrm{FC}$ on day $24(200 \mu \mathrm{L})$ and day $31(0.5$ to $1 \mathrm{~mL}$, terminal $)$ to verify the presence of MM CTCs in the blood. We counted GFP+ cells in drawn blood samples using the green channel of a commercial flow cytometer (Attune NxT, Thermo Fisher). The gating and counting process was as follows: We first performed FC on cultured MM.GFP+ cells in suspension to determine the appropriate side scatter (SSC) and forward scatter (FSC) gates [Fig. 2(a)] and distribution of 530/30 nm blue fluorescence [Fig. 2(b)] for the cells. We used the mode fluorescence intensity of "DG 2" microspheres (Bangs Labs) as a reference and a fluorescence threshold for cell counting, as shown by the dotted red line. Blood samples were preprocessed by first lysing the RBCs with a lysis buffer (420301, Biolegend, San Diego, California) according to the manufacturer's instructions. An example SSC-FSC plot for a blood sample (taken on day 31) is shown [Fig. 2(c)] as well as the blue fluorescence histogram [Figs. 2(d) and 2(e)], wherein most signals were nonfluorescent debris. Application of the threshold (which was defined as the intensity of DG2 microspheres for all samples) allowed us to count MM.GFP + cells. These numbers were divided by the volume of drawn blood to estimate the concentration of MM cells in vivo.

\subsection{Blood Smear Preparation and Imaging}

For mice in $\mathrm{C} 2$, we terminated the experiments on day 36 after injection. The aim was to verify the presence of CTCCs in vivo, which, as we show, was suggested by our DiFC data. We drew and collected $750 \mu \mathrm{L}$ of blood in ethylenediaminetetraacetic acid (EDTA) tubes and euthanized the mice. We created 30 blood smears ${ }^{30}$ per mouse by pipetting $6 \mu \mathrm{L}$ of blood on a glass microscope slide (Fisherbrand Colorfrost microscope slides with clipped corners, Fisher). The cell monolayer area of each slide was imaged with an upright Carl Zeiss microscope with an HXP 120C light source using the bright-field channel and the eGFP channel to detect the presence of MM.GFP+ CTCs and CTCCs. Example images were cropped to $65 \times 65 \mu \mathrm{m}^{2}$ to show example cells and clusters of interest. Overlays were performed using the "merge channels" function in ImageJ.

\subsection{Monte Carlo Simulations}

We used an open-source, GPU-accelerated Monte Carlo program (Monte Carlo eXtreme) to compute the detection sensitivity functions for DiFC. ${ }^{31} \mathrm{We}$ modeled the tail as a homogenous 4-mm diameter, 4-cm long cylinder, with a voxel size of $250 \mu \mathrm{m}^{3}$. We used literature values ${ }^{24}$ for optical properties, including scattering coefficient $\left(\mu_{s}\right)$, absorption coefficient $\left(\mu_{a}\right)$, at the excitation (ex) and emission (em) wavelengths, and the anisotropy coefficient $(g)$. These were as follows: for NIR wavelengths: $\mu_{\text {s-ex }}=22 \mathrm{~mm}^{-1}, \mu_{\text {s-em }}=20 \mathrm{~mm}^{-1}, \mu_{\text {a-ex }}=$ $0.002 \mathrm{~mm}^{-1}, \mu_{\mathrm{a}-\mathrm{em}}=0.0015 \mathrm{~mm}^{-1}, g=0.9$ and for blue-green
(GFP) wavelengths: $\mu_{\mathrm{s} \text {-ex }}=40 \mathrm{~mm}^{-1}, \quad \mu_{\mathrm{s} \text {-em }}=38 \mathrm{~mm}^{-1}$, $\mu_{\mathrm{a}-\mathrm{ex}}=0.02 \mathrm{~mm}^{-1}$, and $\mu_{\mathrm{a}-\mathrm{em}}=0.02 \mathrm{~mm}^{-1}, g=0.9$.

\section{Results}

\subsection{DiFC Performance at Blue-Green Wavelengths}

We built a blue-green DiFC system for use with GFP-expressing cells and transgenic mice models. ${ }^{15,32}$ As shown in Fig. 3, we performed initial testing of the system with reference standard DG intensity level 4 (DG4) fluorescent microspheres in a flow phantom model [Fig. 3(a)]. Example of 5-min sequences of data for PBS (control) and microspheres is shown in Figs. 3(b) and $3(\mathrm{c})$, respectively. As in our prior work, each transient fluorescence "peak" in the data represents a microsphere passing through the DiFC field-of-view.

We anticipated that our GFP-DiFC system might perform poorly at blue-green wavelengths, where light attenuation in biological tissue is relatively high. ${ }^{24}$ Monte Carlo simulations indicated that the expected attenuation with GFP was only $20 \%$ higher than at NIR wavelengths in the $\sim 2-\mathrm{mm}$ DiFC detection depth. As such, optical attenuation was not as serious problem as with whole animal imaging. ${ }^{33}$ Subsequent analysis showed similar modest expected losses with wavelengths corresponding to mCherry and yellow fluorescent protein so that we anticipate that DiFC systems corresponding to these wavelengths could be developed in the future.

We also verified the basic ability of DiFC to detect GFPlabeled cells in vivo immediately after they were introduced into circulation. Example DiFC data taken from the tail ventral vascular bundle [Fig. 3(d)] $30 \mathrm{~min}$ after intravenous injection of PBS (control) and $5 \times 10^{6}$ MM.1s.GFP cells is shown in Figs. 3(e) and 3(f), respectively.

Additional noise from tissue autofluorescence at blue-green wavelengths was another potential concern. However, we tested the GFP-DiFC design in control (sham-injected) SCID mice and found that the background was about $10 \mu \mathrm{A}$ with $20 \mathrm{~mW}$ of power at the sample, which was similar to our NIR system. Likewise, the noise after background subtraction was less than $25 \mathrm{nA}$.

We scanned four control mice, twice weekly with DiFC after the initial sham (PBS) injection, giving 17.5 cumulative hours of DiFC scanning on nontumor bearing mice. We did this as a test of the stability if the DiFC system, i.e., since no GFP+ cells were present, any "detections" were false alarms. On average, we found that the false alarm rate (FAR) was extremely low over all DiFC sessions. The average FAR for arterial-matched CTCs was $0.017 \mathrm{~min}^{-1}$ (or one every $58 \mathrm{~min}$ ) and for CTCCs was $0 \min ^{-1}$ (no false alarms).

\subsection{Disseminated Xenograft Model}

We used an MM DXM, as has been described by our team previously. ${ }^{27}$ MM.1S.Luc.GFP cells were injected i.v. in SCID/ $\mathrm{Bg}$ mice. $\mathrm{MM}$ cells rapidly home to the bone marrow, and then steadily proliferate throughout the skeleton over time. MM cells are eventually observed in circulation in peripheral blood, mimicking the clinical course of the disease. As shown in Figs. 4(a)-4(c), we verified the growth of MM in our mice by weekly BLI. As expected, MM grew in a diffuse pattern in the skeleton, primarily in skull, spine, and hips. Small amounts of BLI above background were observed as early as day 23 with significant disseminated growth by day 30 . 

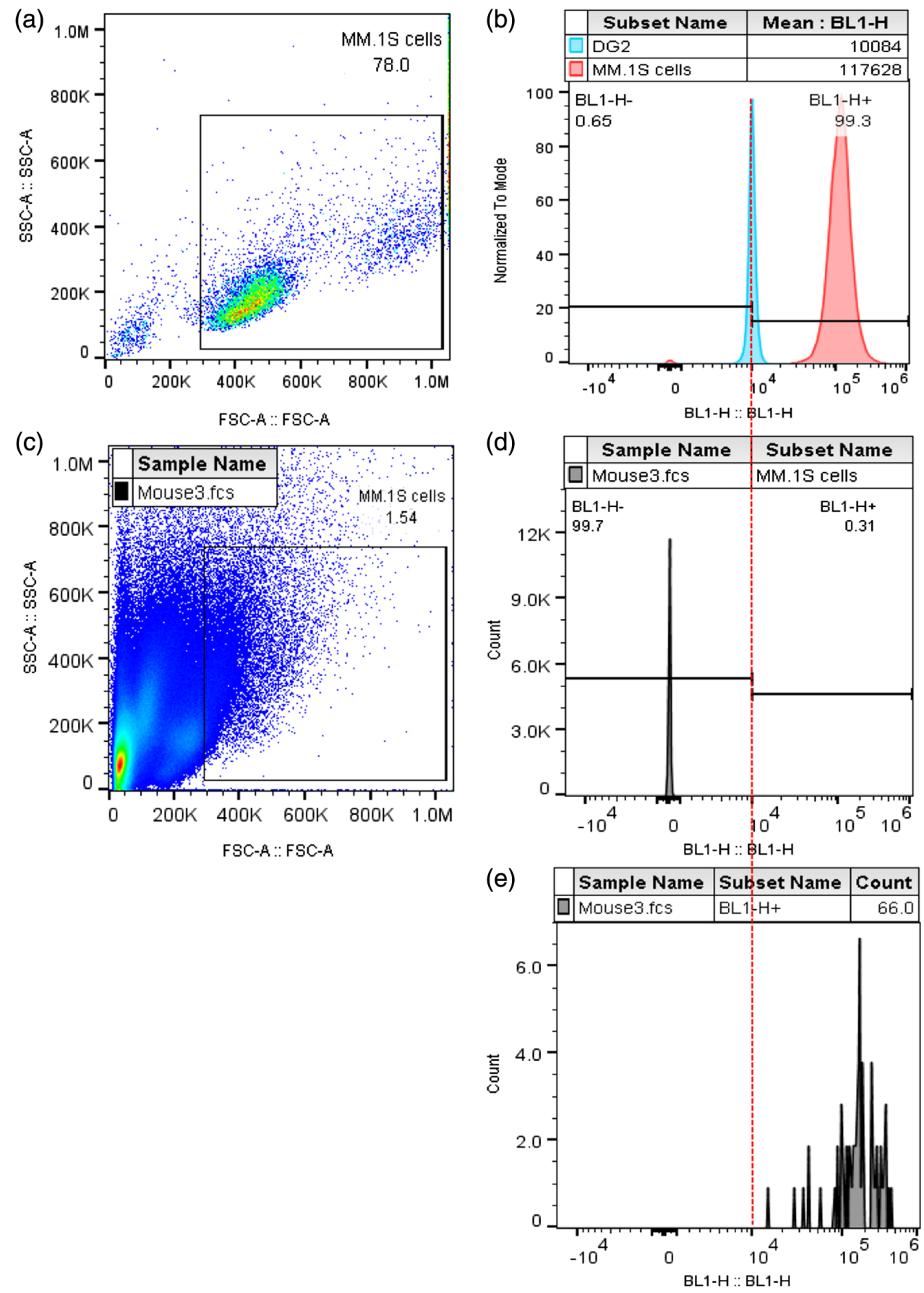

Fig. 2 The FC gating strategy for counting MM.1S.GFP.Luc cells is shown. (a) SSC-FSC plot for MM.1S cells in culture. (b) The blue (BL1) fluorescence of MM.1S.GFP.Luc cells and DG2 microspheres. The mode intensity of DG2 microspheres was used as counting threshold since it was lower than cultured MM.1S.GFP.Luc cells. (c) SSC-FSC for a blood sample drawn from a mouse, with gate shown. RBCs were first depleted using a lysate. (d) Blue (GFP) fluorescence histogram of cells in blood. Most peaks are low-fluorescence debris or unlabeled cells. (e) Blue (GFP) fluorescence histogram of cells exceeding the DG2 threshold only, yielding the cell counts in the sample.

Significant variability in MM growth was observed between mice [Fig. 4(d)], which may have been due to differences in the efficiency of the tail vein injection of MM cells. In one mouse (M3C2), no development of MM was observed by BLI, and there was no sign of BLI signal by the second week (day 8). However, as discussed below, there was also no sign of MM development on DiFC. As such, we chose to keep it in the analysis since it served as an additional blind control.

\subsection{CTC Dissemination During MM Growth}

We scanned the MM-bearing mice with DiFC twice per week after injection. An example 10-min trace from a control mouse is shown in Fig. 5(a) for comparison. Example 10-min sequences of DiFC data from a single mouse (mouse 2, cohort 1; M2C1) from day 14 to day 31 is shown in Figs. 5(b)-5(e). As we showed previously, ${ }^{21} \mathrm{DiFC}$ allows us to distinguish cells moving 
(a)
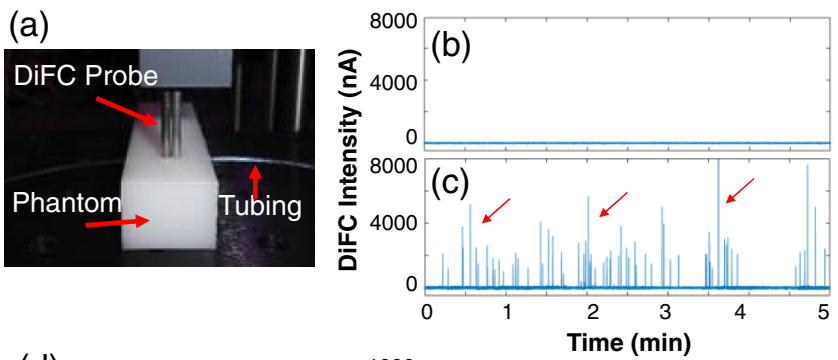

(d)

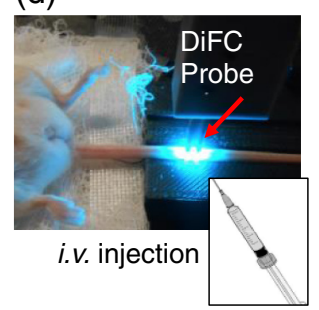

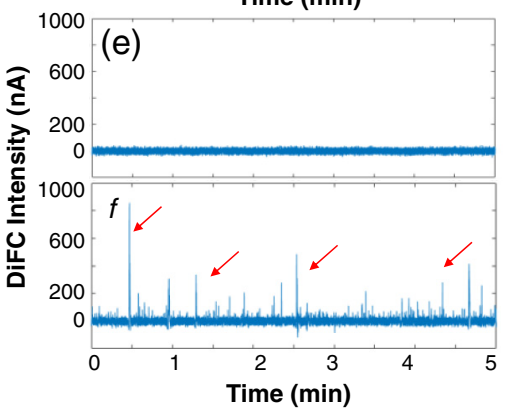

Fig. 3 Validation of the GFP-DiFC system in (a) a flow phantom model with (b) PBS or (c) DG4 fluorescent microspheres. (d) We also verified that the GFP-DiFC system could detect MM.1s labeled cells in vivo. Data from (e) control and (f) MM.1s-injected mice are shown. See text for details.

in the arterial (red markers) and venous (blue markers) directions.

We considered the average (mean) single-cell CTC count rate detected in the arterial matched (VCA) direction during the progression of the disease, as summarized in Fig. 5(f) for all 8 MM-inoculated mice we studied. As shown in Fig. 5(g), small numbers of arterial matched GFP+ CTCs were observed during the first few weeks $\left(<1\right.$ count $\left.\min ^{-1}\right)$. By day 21 , rapid growth in MM CTC numbers was observed in all but one inoculated mouse. This was the same mouse (M3C2) that failed to show the development of MM on BLI, again, most likely due to a failed intravenous injection of cells. As with BLI, significant inter-experimental variability between the mice was observed. However (and shown below), there was a good correlation between BLI and DiFC. For example, mice in cohort 2 generally exhibited both higher BLI intensity and DiFC count rate [inverted triangles, Figs. 4(d) and 5(f)] than cohort 1.

Also, as noted above, we were concerned about transient effects at the start of the scan due to warming of the tail (which was presumed to increase blood flow) by our warming pad. An example of this effect is shown in Fig. 5(h), (M4C1 on day 31) wherein the count rate steadily increased during the first 5 to 10 min of scanning, after which it reached relative steady-state. As such, we removed the first 10 min of data for all the DiFC datasets in this work.

Another interesting feature of the data is that significant short-term variability in the DiFC count rate was observed, with periods of significantly lower or higher detection rates. For example, Fig. 6(a) shows the full 35-min timeline of CTC detections for $\mathrm{M} 2 \mathrm{C} 1$ on day 31 , where each vertical line represents the detection of an arterial-matched cell. Figure 6(b) shows the mean count rate for the scan (red line; $7.7 \mathrm{~min}^{-1}$ ) and the moving average over 60-s intervals (blue dotted line), where the count rate varied from 0 to $22 \mathrm{~min}^{-1}$ during scanning. This significant variability was always observed for the mice in this study and may be expected in part from statistical effects,
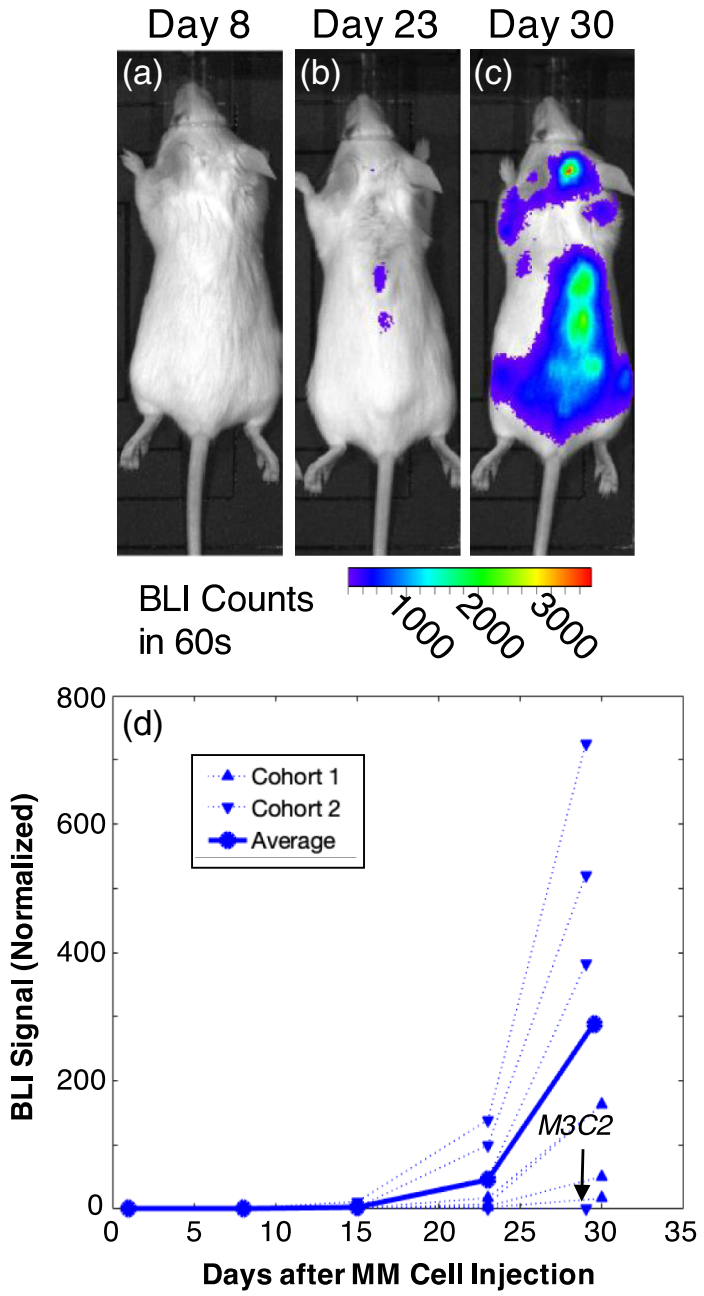

Fig. 4 We performed BLI imaging weekly for all mice for 1 month following tail vein injection of MM.1S.GFP.Luc cells. (a)-(c) Luminescence increased as the MM disease grew in the bone marrow, which was first observable in small areas of (b) the spine by day 23. (c) By day 30, diffuse patterns of MM growth were observable in the skull, spine, and hips. (d) This general pattern was observed for all inoculated mice except for one (M3C2), with significant interexperimental variability between mice.

particularly with small-time samples and rare cells. ${ }^{12,13}$ The implications of this are discussed in more detail below.

DiFC also allowed us to measure the speed of cells in the arterial direction by analyzing the arrival time between the two fiber probes, which were separated by $3 \mathrm{~mm}$ [Fig. 1(d)]. For these mice, the average speed of cells in the arterial direction was $26.5 \mathrm{~mm} \mathrm{~s}^{-1}$. We note that this was significantly slower than we measured in nude mice previously, ${ }^{21}$ where the average arterial cell speed was $112 \mathrm{~mm} \mathrm{~s}^{-1}$. We attribute the difference to the physically smaller size of the tails (and presumably VCA) of SCID/Bg strain compared to nude mice. The measured peak width for cells in the arterial direction was $39 \mathrm{~ms}$ in SCID mice, which is consistent with the slower speed, i.e., slower-moving cells take longer to move through the $\sim 1$-mm DiFC field-ofview.

\subsection{CTC Clusters}

Interestingly, DiFC scanning also revealed the presence of large, irregular-shaped signal features, such as those shown in 

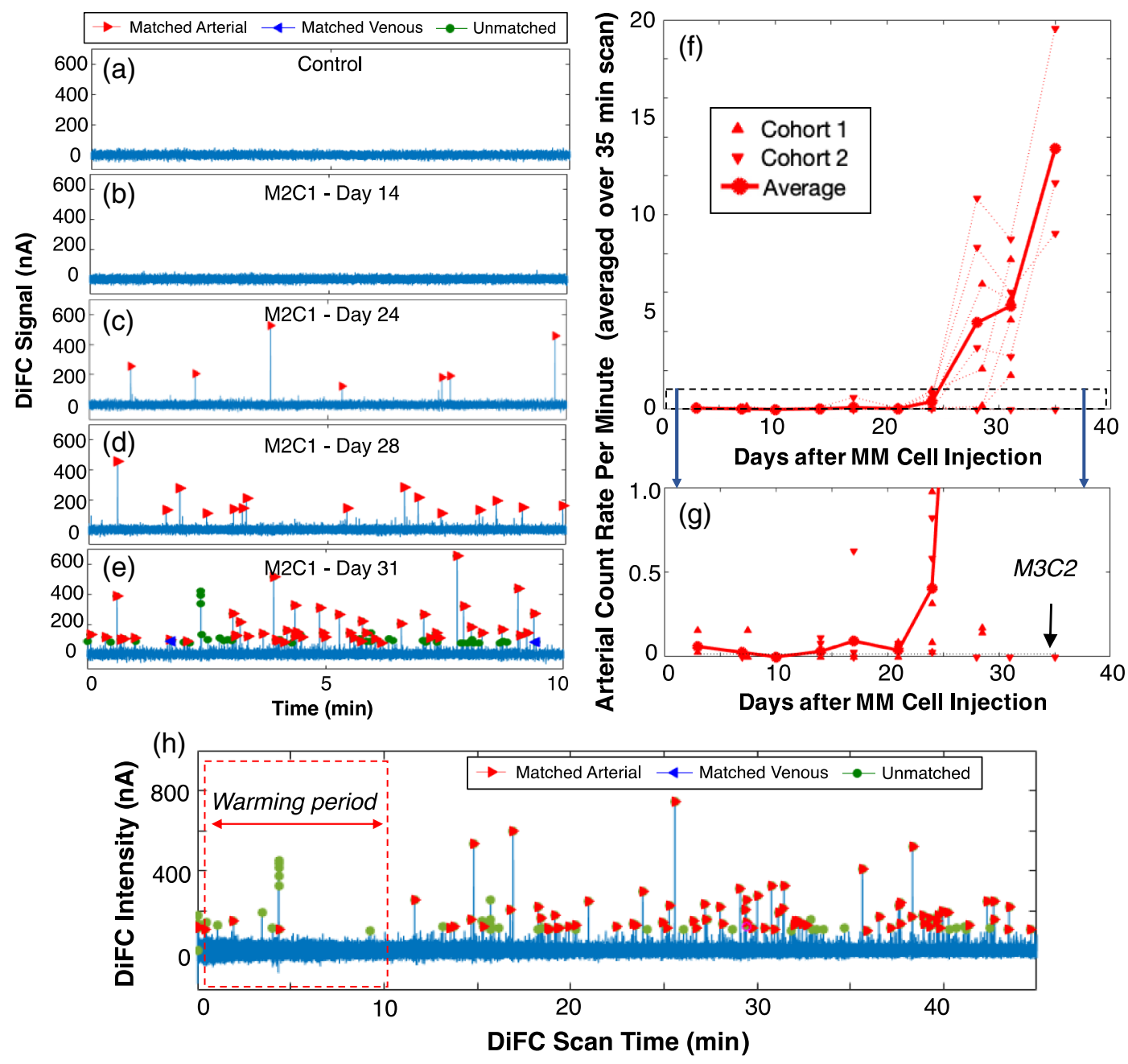

Fig. 5 We performed DiFC scanning twice per week during the development of the xenograft model. (a) An example 10-min DiFC sequence from a control (PBS injected) mouse is shown, illustrative of the low FAR of the DiFC system. (b)-(e) As the MM disease progressed, GFP+ MM cells were observable in circulation with increasing frequency. (f) The mean single-cell count rate in the arterial direction for all mice is shown, showing growth over the course of the disease, as well as significant interexperimental variability. (g) An expanded view of (f) for mean DiFC-count rates between 0 and 1 counts $\mathrm{min}^{-1}$. DiFC allowed detection of very low numbers of circulating cells above the FAR (dotted line, $0.016 \mathrm{~min}^{-1}$ ). As with BLI, one mouse (M3C2) failed to show signs of MM growth. (h) A transient increase in DiFC count was frequently observed in the first 5 to $10 \mathrm{~min}$ of scanning, which we attribute to warming of the mouse tail and corresponding increase in blood flow.
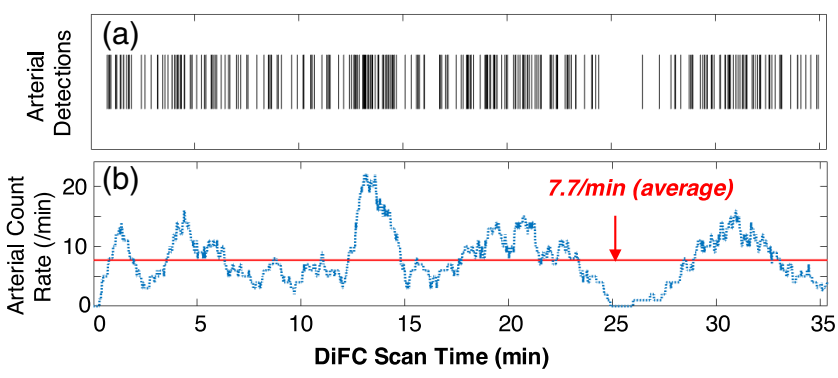

Fig. 6 (a) Raster plot showing the detection times of arterial-matched CTCs for mouse M2C1 on day 31 during DiFC scanning, where each vertical line represents one detected cell. (b) The average count rate for this scan was $7.7 \mathrm{~min}^{-1}$ (red line), but significant variability was observed when considering a 60 -s moving average window (blue dotted line).
Figs. 7(a)-7(d). These were significantly wider (temporally) and higher (amplitude) than individual matched cells. We hypothesized that these signals originated from MM CTCCs in the blood. Specifically, we used the criteria of at least 100-ms peak FWHM and 300-nA amplitude for identification of CTCCs. The height and width profiles of single-CTCs and CTCCs are shown in Fig. 7(e), showing good separation between the two groups. As shown in Fig. 7(f), these CTCC-like signals were observed soon after single CTCs were detectable (day 24) and appeared with increasing frequency as the disease progressed to a maximum of about $2 \mathrm{~min}^{-1}$. We also note that these were not artifactual since signals of this type were never observed in our control mice.

We analyzed the pulse amplitude of the CTCC-like signals relative to single peaks and estimated the equivalent number of cells in clusters, as shown in Fig. 7(g). Using this, most MM 

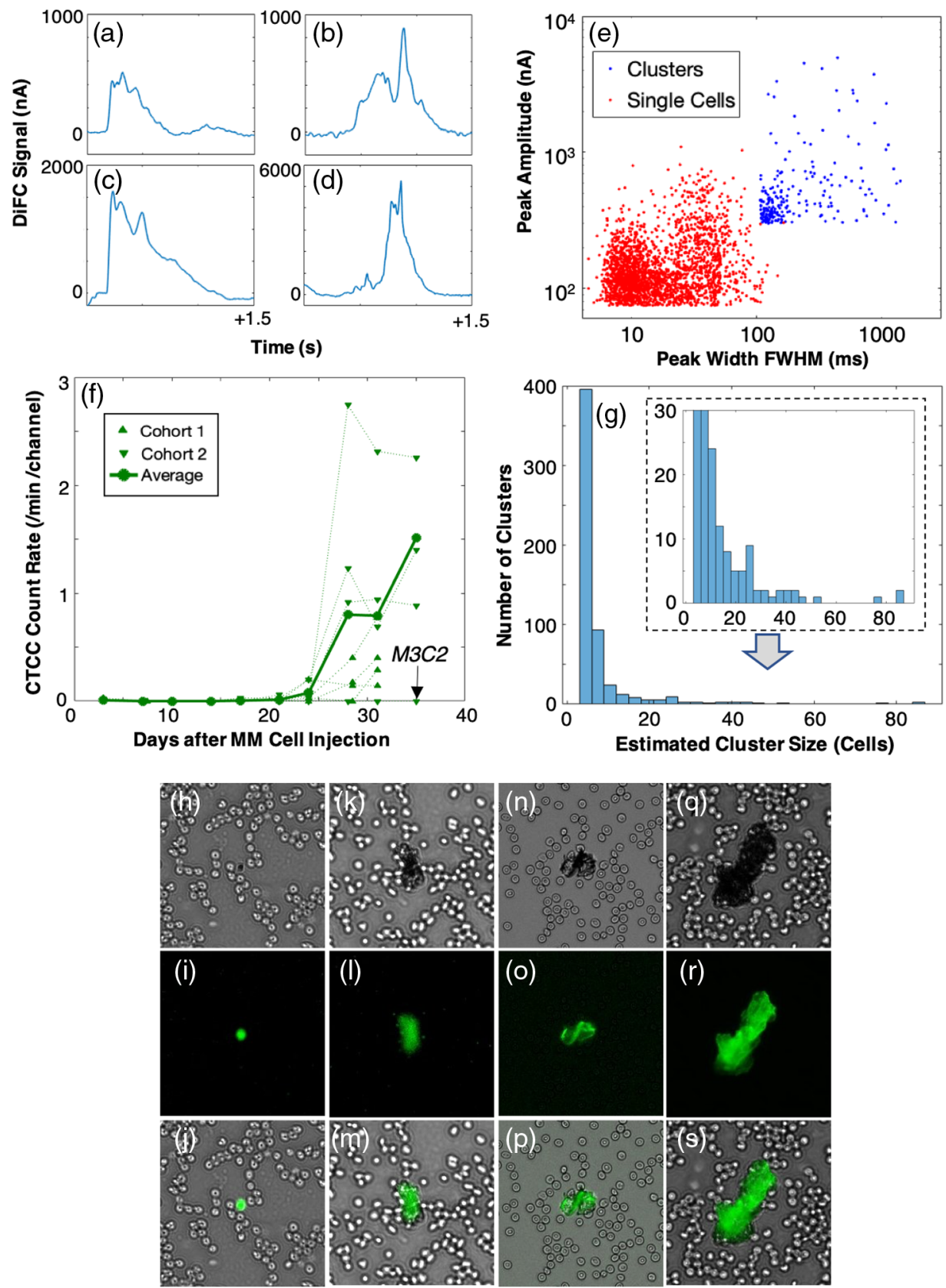

Fig. 7 (a)-(d) DiFC also showed large, irregularly shaped signals (relative to individual cells), which we hypothesized were due to the presence of CTCCs. (e) We used a threshold of 300-nA amplitude and 100-ms peak width to identify CTCCs. (f) These were observed with MM-injected mice only (never controls) and appeared approximately as soon as single CTCs were observed in circulation at a rate of $\sim 10 \%$ of the arterial count rate. (g) Analysis of the peak amplitudes allowed us to estimate the sizes of the clusters, which were frequently fewer than 10 cells, but individual (inset) clusters were very large, with dozens of cells. (h)-(s) Example white-light, GFP fluorescence, and merged (overlay) blood smear micrographs from MM.1S bearing mice sacrificed on day 36 after injection. Most observed MM GFP + cells were single cells $(h)-(j)$, but small numbers of CTCCs $(k)-(s)$ were also observed as predicted from our DiFC measurements. Each image is $65 \times 65 \mu \mathrm{m}^{2}$.

CTCCs were usually estimated to be smaller than 10 cells, with mean and median estimated sizes of 6.4 and 4 cells, respectively. However, a small number of large CTCC-like peaks were also observed with estimated sizes approaching 100 cells [Fig. 7(g), inset]. We did not use the width of the peak in estimation of the CTCC sizes since, unlike microscopy-IVFC, ${ }^{34}$ even large clusters of cells are much smaller than the $\sim 1-\mathrm{mm}$ DiFC field-of-view. Therefore, we attribute the broad temporal width of CTCCs relative to individual CTCs to significantly slower speed of motion in the blood vessel, as opposed to their physical size. In addition, unlike single cells, there were frequently relatively long intervals between detection on the two channels, implying slow speed (see Sec. 4).

CTCCs have not been reported in blood for this MM.1S xenograft model previously. Moreover, we were able to identify only one direct report of clusters in the MM literature, where 
clusters were detected in patient blood samples. ${ }^{35}$ Therefore, we performed a secondary check for the presence of clusters in the blood by GFP imaging of blood smears. ${ }^{30}$ This technique allowed us to form a smooth cell monolayer using small (6 $\mu \mathrm{L}$ ) blood samples. These were taken from the second cohort of xenograft mice (C2) that were euthanized 36 days after injection. Clusters are simply defined as "two or more tumor cells that travel together in the blood" and may form prior to shedding or while in circulation. ${ }^{16,18,35}$ Since only MM.1s cells express GFP in our mice, multicellular groupings of GFP+ cells in blood samples allowed us to positively identify the presence of clusters.

Example fluorescence micrograph images of the blood smears are shown in Figs. 7(h)-7(s). Although the vast majority of observed MM.GFP+ cells on the blood smears were individual CTCs [Figs. 7(h)-7(j)], we did observe a small number of CTCCs of varying size from a fewer than 10 [Figs. 7(k)-7(p)] to dozens or hundreds of cells [Figs. 7(q)-7(s)]. The blood smear imaging data did not permit accurate estimation of the numbers of CTCs in the clusters, but the observed physical sizes were in good general agreement with our DiFC-derived estimates [Fig. 7(g)].

It is very unlikely that these multi-cellular groupings formed on the slides due to the low numbers of MM cells in the blood (see below) and since the blood smear preparation is a standard technique that is known to produce a cell monolayer. ${ }^{30} \mathrm{We}$ also used anticoagulants (EDTA) to prevent this, and we did not observe multi-cellular groupings of this type for any other erythrocytes or leukocytes in the blood. We also note that, in general, clusters may (and frequently do) contain noncancer cells such as immune or epithelial cells ${ }^{18}$ so that GFP- cells may also be observed in the groupings. We plan to further characterize these in the future using additional cell surface markers on the blood smears, for example, for epithelial, mesenchymal, and immune cell markers. In summary, these findings verify that MM.1S does circulate in clusters in this xenograft model, to our knowledge, for the first time.

\subsection{Estimation of CTC Numbers from DiFC Measurements}

Finally, we were interested in calibrating our DiFC measurements to the actual CTC number in blood. First, we calculated the estimated "corrected" equivalent DiFC count rate per minute, as shown in Fig. 8(a). To do this, we summed the contributions from individual cells [Fig. 7(g)] with the contributions from the detected CTCCs, which were derived from the cluster size estimates and the average detection rate.

We then estimated the CTC number in peripheral blood (in cells $\mathrm{mL}^{-1}$ ) from our measured $\mathrm{DiFC}$ count rate (in counts $\mathrm{min}^{-1}$ ) in two ways as follows. First, we used the average cell speed in the tail artery measured by DiFC of $v_{s}=26.5 \mathrm{~mm} \mathrm{~s}^{-1}$. Making the simplifying assumption that the tail artery was a cylinder with 200- to 250- $\mu$ m diameter with homogenous flow, this implies that the blood flow rate through DiFC in the artery was about 0.8 to $1.3 \mu \mathrm{L} \mathrm{s}^{-1}$ or 50 to $78 \mu \mathrm{L} \mathrm{min}^{-1}$.

Second, in one cohort of mice (C1), we drew $200 \mu \mathrm{L}$ of blood on day 24 , and 0.5 to $1 \mathrm{~mL}$ of blood on day 31 (terminal). GFP-labeled MM cells in the blood samples were counted by FC, as shown in Fig. 8(b). On day 24, we were unable to detect any GFP-labeled cells in the blood samples by FC, whereas on day 31, small numbers of GFP+ CTCs were detectable. The average number of GFP + cells in the four mice studies was
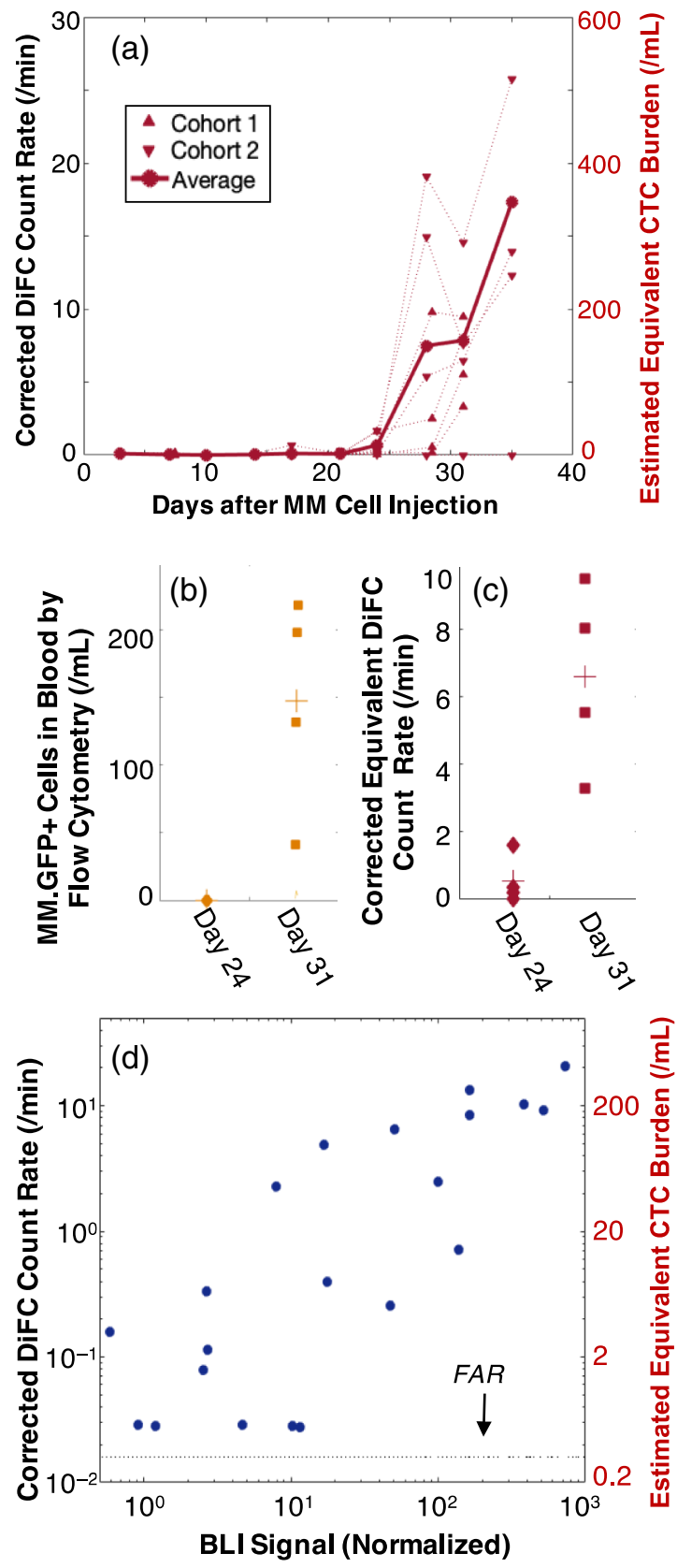

Fig. 8 (a) We calculated the "corrected" DiFC count rate, which combined contributions from single CTCs and CTCCs (see text for details). (b) We drew blood samples on days 24 and 31 and counted MM GFP+ cells in the blood. (c) We compared these to the DiFC count rate on the same days, allowing us to estimate the detection sensitivity of DiFC. (d) The DiFC count rate also linearly correlated with MM disease burden measured by BLI for all mice and time-points $\left(r^{2}=0.75\right)$. All indicated points are above the FAR of the system (black dotted line).

147 cells $\mathrm{mL}^{-1}$ of blood, for which we assume an uncertainty of approximately $10 \%\left( \pm 15 \mathrm{CTCs} \mathrm{mL}^{-1}\right)$ due to uncertainty in the exact volume of blood drawn and analyzed. The multiple steps of blood sample preparation including centrifugation and RBC lysing are expected to break-up any CTCCs in the blood. We also previously showed that this method of enumerating CTCs does not cause measurable loss of CTCs due to handling. ${ }^{21}$ We compared the estimates of CTC numbers in the blood to the corrected equivalent DiFC count rate from the same 
mice on the same days [Fig. 8(c)], which was on average 6.6 counts $\mathrm{min}^{-1}$. Dividing the two yielded an estimated sampling rate of $45 \pm 4 \mu \mathrm{L} \mathrm{min}^{-1}$.

The two methods of estimation were in good general agreement, which is reasonable considering the substantial uncertainty involved in both. It is possible that our method for estimating CTCs in blood from DiFC (including our threshold and signal processing method) underestimates the true numbers in blood. It is also possible that DiFC does not detect the most weakly labeled GFP + cells. Refining our signal processing algorithm and instrument is an ongoing area of work in our group. However, we chose a conservative (round) value of $50 \mu \mathrm{L} \mathrm{min}^{-1}$ for estimation of CTC burden on the right axis of Fig. 8(a). We note that this is lower than the previous sampling rate we estimated with our NIR DiFC system in nude mice ${ }^{21}$ which we attribute to the smaller tail in SCID mice and correspondingly lower measured blood flow speed. Compared to other IVFC methods, this high blood sampling rate makes DiFC particularly well suited to studying rare circulating cells in the range of fewer than 100 cells $\mathrm{mL}^{-1}$.

Our data were marked by the presence of significant interexperimental biological variability observed in Figs. 4, 5, and 7, even in nominally identical experimental repeats with inbred mice and a cultured MM cell line. However, the same variability was measured on both systems for specific mice at specific times. Specifically, the overall tumor burden in the mice measured by BLI overall correlated well with the DiFC count rate. These data are shown for all points taken in this study in Fig. 8(d), where a good linear correlation was observed $\left(r^{2}=0.75\right)$. This underscores the value of longitudinal study of individual mice using noninvasive diagnostic instruments, such as DiFC.

\section{Discussion and Conclusions}

We previously reported our fiber bundle ${ }^{29}$ and DiFC design ${ }^{21}$ and showed that it was capable of detecting extremely rare circulating cells in phantoms in vitro and in mice in vivo without having to draw blood samples. In this work, we developed a GFP-compatible DiFC system and demonstrated its first use with an MM xenograft model in mice. As we noted previously, the detection sensitivity of blue-green $\mathrm{DiFC}$ - with respect to the minimum detectable fluorescent-labeling of target cellsis expected to be lower than microscopy-IVFC, which uses confocal light detection. ${ }^{10}$ However, in ongoing studies in our lab, we have tested a number of GFP-labeled cell lines obtained from commercial and academic sources in nude and SCID mice, which thus far are sufficiently bright for detection with DiFC.

To reiterate, the main advantage of $\mathrm{DiFC}$ versus microscopyIVFC methods is the use of diffuse light, which allows sampling of at least an order of magnitude greater circulating blood volumes. In this model, we estimated the sampling rate of $50 \mu \mathrm{L} \mathrm{min}^{-1}$, whereas microcopy IVFC samples on the order of $1 \mu \mathrm{L} \mathrm{min}^{-1}$. Prior work using microscopy-IVFC to study CTCs in an orthotopic tumor model in mice showed detection of a few cells per hour ${ }^{36}$ or at concentrations in the range of $10^{4}$ cells $\mathrm{mL}^{-1}$. $^{37}$

By contrast, in this study, the average count rate on the first day of detection (day 17 or 21, depending on the mouse) was 0.2 counts $\mathrm{min}^{-1}$, which is equivalent to an estimated CTC burden of only 1 cell $\mathrm{mL}^{-1}$ of blood. The lowest count rates above the FAR (e.g., 0.03 counts $\mathrm{min}^{-1}$ for $\mathrm{M} 3 \mathrm{C} 1$, day 24) were equivalent to $\sim 0.4$ cells $\mathrm{mL}^{-1}$ or about 1 cell in the entire peripheral blood volume of the mouse. These results demonstrate that DiFC is able to detect extremely rare circulating cells at early stages of MM growth.

Such low CTC numbers are difficult to detect with liquid biopsy techniques involving nonterminal drawn blood samples. As illustrated in Fig. 6, we also observed significant variability in the CTC count rate in short-term (minute-to-minute) intervals. Consideration of the count rate in a short 60-s time interval is analogous to estimating the CTC burden from a small $(\sim 50 \mu \mathrm{L})$ blood sample, which, in this case, could vary by more than a factor of 3 in the span of only $35 \mathrm{~min}$. This was observed over all mice we studied with DiFC in these experiments. The shortterm kinetics and statistics of CTC detection are the subject of ongoing work in our group.

A result in this work was the measurement of highamplitude, temporally wide peaks, which we attributed to the presence of CTCCs and subsequently verified by fluorescence microscopic imaging of blood smears. Measurement of CTCCs in this model with DiFC was not anticipated based on our previous work with IVFC but was not surprising given that MM has been shown in a small number of reports to form clusters in the bone marrow ${ }^{27,28}$ and in at least one previous report in patient blood. ${ }^{35}$ In addition, CTCCs have been reported for many other cancer types. However, very little is known about MM CTCCs, such as their composition, size, and in vivo kinetics. As others have noted, this is in part because most of methods of CTC isolation are not designed to capture clusters. ${ }^{18}$ While we interpret the data from the xenograft model with some caution, there were nevertheless some interesting features of our data:

- CTCCs were consistently detected in circulation almost as soon as individual CTCs were observed in circulation (on day 21 to 24), suggesting that CTCCs shed with approximately the same kinetics.

- CTCCs were observed with about $10 \%$ of the frequency of individual CTCs. This is in good general agreement with other literature reports of CTCCs, such as in breast cancer $^{20}$ and suggests that while CTCCs are rare, they are also not uncommon. The ratio of CTCs to CTCCs was approximately consistent throughout the studies.

- According to DiFC data and fluorescence microscopic imaging estimates, most CTCCs were smaller than 10 cells. A literature search failed to reveal other published estimates of cluster sizes specifically for MM.1S, or for MM in general. However, these values are in good agreement with literature studies of CTCC sizes for other cancers in mice and in humans. ${ }^{18}$

- CTCCs appeared to move slowly relative to single CTCs. Cluster signals on DiFC were consistently much temporally wider - the average FWHM pulse-width of single CTCs was $~ 39 \mathrm{~ms}$, whereas clusters were on average $320 \mathrm{~ms}$, and in some cases, longer than a second.

In summary, DiFC is a powerful small animal research tool that can provide useful complementary information about in vivo CTC and CTCC behavior for liquid biopsy assays. For example, kinetics of CTC and CTCC shedding can be measured with DiFC to inform the timing of blood draws so that more detailed molecular and genetic analysis of cells can be performed. As we have noted previously, although DiFC can potentially be applied in larger limbs and species, it also relies on the use of fluorescence contrast, which makes translation to 
human use challenging. Therefore, we view DiFC primarily as a small animal research tool in the foreseeable future.

Ongoing work in our group is exploring the use of DiFC in other models of animal models of metastasis, including a Lewis lung carcinoma and SKOV-3 ovarian cancer and their response to anticancer therapeutics. We plan to perform a more detailed characterization of the observed clusters in the future. We are also studying the use of DiFC with targeted fluorescent molecular contrast agents, such as folate-targeted probes that have been shown to have high-affinity and specificity for CTCs. ${ }^{38}$ Moreover, we anticipate that DiFC could have significant utility with other studies involving other noncancer circulating cell types as well as circulating fluorescent nanosensors.

\section{Disclosures}

The authors have no relevant financial interests in this article and no potential conflicts of interest to disclose.

\section{Acknowledgments}

This work was funded by the National Institutes of Health (Grant No. R01HL124315; NHLBI). We thank Eric Marple of EMVision LLC for helpful advice and discussion related to the fiber probe design. We thank Prof. Qianqian Fang (Northeastern University) for assistance using the MCX Monte Carlo software package. The datasets generated and analyzed during the current study, as well as the analysis code (written in MATLAB) are available from $\mathrm{MN}$ on a reasonable request.

\section{References}

1. P. S. Steeg and D. Theodorescu, "Metastasis: a therapeutic target for cancer," Nat. Clin. Pract. Oncol. 5(4), 206-219 (2008).

2. F.-C. Bidard et al., "Clinical validity of circulating tumour cells in patients with metastatic breast cancer: a pooled analysis of individual patient data," Lancet Oncol. 15(4), 406-414 (2014).

3. S. A. Joosse, T. M. Gorges, and K. Pantel, "Biology, detection, and clinical implications of circulating tumor cells," EMBO Mol. Med. 7(1), 1-11 (2015)

4. K. Pantel and M. R. Speicher, "The biology of circulating tumor cells," Oncogene 35(10), 1216-1224 (2016).

5. J. Massague and A. C. Obenauf, "Metastatic colonization by circulating tumour cells," Nature 529(7586), 298-306 (2016).

6. B. Hong and Y. $\mathrm{Zu}$, "Detecting circulating tumor cells: current challenges and new trends," Theranostics 3(6), 377-394 (2013).

7. G. Theil et al., "The use of a new cellcollector to isolate circulating tumor cells from the blood of patients with different stages of prostate cancer and clinical outcomes - A proof-of-concept study," PLoS One 11(8), e0158354 (2016).

8. S. M. Azarin et al., "In vivo capture and label-free detection of early metastatic cells," Nat. Commun. 6, 8094 (2015).

9. V. V. Tuchin, A. Tarnok, and V. P. Zharov, "In vivo flow cytometry: a horizon of opportunities," Cytometry A 79(10), 737-745 (2011).

10. C. Hartmann et al., "Fluorescence detection, enumeration and characterization of single circulating cells in vivo: technology, applications and future prospects," Phys. Med. Biol. 63(1), 01TR01 (2017).

11. J. Hoff, "Methods of blood collection in the lab mouse," Lab Anim. 29(10), 49-53 (2000).

12. A. L. Allan and M. Keeney, "Circulating tumor cell analysis: technical and statistical considerations for application to the clinic," J. Oncol. 2010, 1-10 (2010).

13. Z. S. Lalmahomed et al., "Circulating tumor cells and sample size: the more, the better," J. Clin. Oncol. 28(17), e288-e289; author reply e290 (2010).

14. K. H. Wong et al., "The role of physical stabilization in whole blood preservation," Sci. Rep. 6, 21023 (2016).

15. C. M. Pitsillides et al., "Cell labeling approaches for fluorescence-based in vivo flow cytometry," Cytometry A 79(10), 758-765 (2011).
16. J. M. Hou et al., "Clinical significance and molecular characteristics of circulating tumor cells and circulating tumor microemboli in patients with small-cell lung cancer," J. Clin. Oncol. 30(5), 525-532 (2012).

17. D. G. Duda et al., "Malignant cells facilitate lung metastasis by bringing their own soil," Proc. Natl. Acad. Sci. U. S. A. 107(50), 21677-21682 (2010).

18. Y. Hong, F. Fang, and Q. Zhang, "Circulating tumor cell clusters: what we know and what we expect (review)," Int. J. Oncol. 49(6), 2206-2216 (2016).

19. M. Giuliano et al., "Perspective on circulating tumor cell clusters: why it takes a village to metastasize," Cancer Res. 78(4), 845-852 (2018).

20. N. Aceto et al., "Circulating tumor cell clusters are oligoclonal precursors of breast cancer metastasis," Cell 158(5), 1110-1122 (2014).

21. X. Tan et al., "In vivo flow cytometry of extremely rare circulating cells," Sci. Rep. 9, 3366 (2019).

22. I. Georgakoudi et al., "In vivo flow cytometry: a new method for enumerating circulating cancer cells," Cancer Res. 64(15), 5044-5047 (2004).

23. Y. He et al., "In vivo label-free photoacoustic flow cytography and on-the-spot laser killing of single circulating melanoma cells," Sci. Rep. 6, 39616 (2016)

24. S. L. Jacques, "Optical properties of biological tissues: a review," Phys. Med. Biol. 58(11), R37-R61 (2013).

25. X. Shu et al., "Mammalian expression of infrared fluorescent proteins engineered from a bacterial phytochrome," Science 324(5928), 804-807 (2009).

26. N. C. Shaner, P. A. Steinbach, and R. Y. Tsien, "A guide to choosing fluorescent proteins," Nat. Methods 2(12), 905-909 (2005).

27. J. M. Runnels et al., "Optical techniques for tracking multiple myeloma engraftment, growth, and response to therapy," J. Biomed. Opt. 16(1), 011006 (2011).

28. I. M. Ghobrial, "Myeloma as a model for the process of metastasis: implications for therapy," Blood 120(1), 20-30 (2012).

29. V. Pera et al., "Diffuse fluorescence fiber probe for in vivo detection of circulating cells," J. Biomed. Opt. 22(3), 037004 (2017).

30. B. Houwen, "Blood film preparation and staining procedures," Clin. Lab Med. 22(1), 1-14 (2002).

31. L. Yu et al., "Scalable and massively parallel Monte Carlo photon transport simulations for heterogeneous computing platforms," J. Biomed. Opt. 23(1), 010504 (2018).

32. R. Y. Tsien, "The green fluorescent protein," Аnnu. Rev. Biochem. 67, 509-544 (1998)

33. N. C. Deliolanis et al., "In vivo tomographic imaging of red-shifted fluorescent proteins," Biomed. Opt. Express 2(4), 887-900 (2011).

34. Y. Suo et al., "Proportion of circulating tumor cell clusters increases during cancer metastasis," Cytometry A 91(3), 250-253 (2017).

35. L. Zhang et al., "Detection and characterization of circulating tumour cells in multiple myeloma," J. Circ. Biomark 5, 10 (2016).

36. Z. C. Fan et al., "Real-time monitoring of rare circulating hepatocellular carcinoma cells in an orthotopic model by in vivo flow cytometry assesses resection on metastasis," Cancer Res. 72(10), 2683-2691 (2012).

37. D. Hwu et al., "Assessment of the role of circulating breast cancer cells in tumor formation and metastatic potential using in vivo flow cytometry," J. Biomed. Opt. 16(4), 040501 (2011).

38. W. He et al., "Quantitation of circulating tumor cells in blood samples from ovarian and prostate cancer patients using tumor-specific fluorescent ligands," Int. J. Cancer 123(8), 1968-1973 (2008).

Roshani Patil received her BS degree in biomedical engineering from the Illinois Institute of Technology in 2015, and her MS degree in bioengineering from Northeastern University in 2019.

Xuefei Tan received her BS degree from Nankai University in 2010 and her PhD in the Department of Electrical and Computer Engineering at Northeastern University in 2019.

Mark Niedre received his PhD from the University of Toronto in the Department of Medical Physics in 2004. He is an associate professor of bioengineering at Northeastern University and a senior member of SPIE.

Biographies of the other authors are not available. 